\title{
Mothers' infant feeding experiences: constraints and supports for optimal feeding in an HIV-impacted urban community in South Africa
}

\author{
Lindiwe Sibeko', Anna Coutsoudis², S'phindile Nzuza ${ }^{2}$ and Katherine Gray-Donald ${ }^{1, *}$ \\ ${ }^{1}$ School of Dietetics and Human Nutrition, McGill University, 21,111 Lakeshore Road, Ste. Anne de Bellevue, \\ Quebec, Canada, H9X 3V9: ${ }^{2}$ University of KwaZulu-Natal, Department of Paediatrics \& Child Health, Doris \\ Duke Medical Research Institute, Nelson R Mandela School of Medicine, 710 Umbilo Road, Durban 4001, \\ South Africa
}

Submitted 14 November 2007: Accepted 7 January 2009: First published online 27 March 2009

\begin{abstract}
Objective: To better understand the enabling and challenging factors impacting on infant feeding practices in communities with a high HIV prevalence.

Design: Qualitative study, with data collected through in-depth interviews and observations of mothers, in addition to discussions with health-service providers. Setting: Urban settlement in the province of KwaZulu-Natal, South Africa.

Subjects: Mothers recruited from an HIV clinic and from within the community. Results: Emerging from discussions with mothers on the acceptability of alternative feeding methods were the challenges they encountered in feeding their infants. Mothers readily identified feeding in the context of HIV infection as an issue of great concern, encompassing three central themes: (i) stigma and disclosure of HIV; (ii) confusion and coercion; and (iii) diarrhoea, sickness and free formula. It became evident that mothers rarely received quality infant feeding counselling and consequently mixed feeding, a widespread practice but one that is highly risky for HIV transmission, remained a common feeding practice. Exclusive breast-feeding (EBF) was best practised with support, following disclosure of HIV status. Availability of free formula did not guarantee exclusive formula feeding but instead led to inappropriate feeding practices.

Conclusions: In addition to providing accurate information, health-care workers must be empowered to counsel mothers effectively, addressing issues of disclosure and thereby facilitating mobilization of maternal support networks. These findings illustrate the challenges that exist in policy translation within the context of quality of training for health-care workers on optimizing maternal infant feeding practices, particularly in HIV-prevalent, resource-poor settings.
\end{abstract}

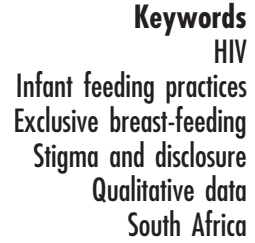

The African continent is home to approximately 22 million adults and children living with HIV/AIDS ${ }^{(1)}$, substantially burdening an already marginalized region of the world to bear the brunt of the global HIV epidemic. Furthermore, by the end of 2006, paediatric infections accounted for an estimated $2 \cdot 3$ million of the world's children living with HIV, $90 \%$ of whom are infected through vertical transmission of the virus ${ }^{(2)}$. An estimated 280000 South African children under 15 years of age live with $\mathrm{HIV}^{(1)}$, the majority infected through mother-to-child transmission (MTCT). Hence, on a national level, prevention of MTCT (PMTCT) is considered a public health concern of high priority.

Non-exclusive breast-feeding is associated with the infection of about 300000 children annually ${ }^{(3)}$; however, breast-feeding in resource-poor countries is a powerful child survival tool with the potential of preventing an estimated $13 \%$ of child deaths from malnutrition and non-HIV common childhood infections ${ }^{(4)}$. Substantial evidence now exists illustrating the role of exclusive breast-feeding (EBF) in minimizing transmission risk of HIV while promoting child health, resulting in greater HIV-free child survival ${ }^{(5-7)}$. Recently, an African-based prospective study with feeding data on 1276 infants found a low transmission risk (4\%) following 6 months of EBF, with mortality at 3 months more than double in formula-fed babies compared with EBF infants ${ }^{(8)}$.

Although infant formula is the recommended feeding option for HIV-positive mothers in developed countries ${ }^{(9)}$, this is rarely feasible or a preferred choice for women living in resource-poor communities due to the prohibitive cost of formula, lack of safe water, lack of infrastructure to ensure 
Table 1 Characteristics and type of HIV status disclosure identified by motivation and approach

\begin{tabular}{lcl}
\hline & Type of disclosure & \multicolumn{1}{c}{ Disclosure characteristics } \\
\hline Intention of disclosure & Voluntary & Decision to reveal HIV status without coercion \\
& Involuntary & HIV status is divulged unintentionally or without consent \\
Disclosure approach & Direct & Straightforward approach to revealing status \\
& Proxy & HIV status is implied through action or association, not openly revealed
\end{tabular}

Adapted from Varga et al. ${ }^{(12)}$.

consistent formula availability, socio-cultural values and the stigma associated with formula feeding. Hence, under conditions where replacement feedings are not acceptable, feasible, affordable, safe or sustainable (AFASS), WHO/ UNAIDS/UNICEF ${ }^{(10)}$ guidelines encourage mothers living with HIV to breast-feed exclusively for 6 months; at which point breast-feeding cessation is only recommended if a replacement milk compliant to AFASS conditions is available, to be combined with complementary foods. Operational interpretation of these guidelines requires counselling of mothers by trained health-care staff. Lack of disclosure of HIV status can serve as a significant impediment to maternal implementation of these optimal infant feeding guidelines. Despite the fear of discrimination associated with disclosure, mothers living with HIV who disclose their status have been found to experience positive outcomes ${ }^{(11)}$. Varga et $a l^{(12)}$ view disclosure as a dynamic process, as outlined in Table 1.

Translation and implementation of infant feeding policy guidelines is essential for functional PMTCT programmes; consequently gaining insight into the real experiences and challenges of mothers who are recipients of infant feeding information is crucial feedback for programme development.

In the present paper we report on data that emerged from a qualitative study aimed primarily at examining the receptivity of mothers and other community members to the use of heat-treated expressed breast milk as an alternative feed (L Sibeko, A Coutsoudis, S Nzuza and $\mathrm{K}$ Gray-Donald, unpublished results). Issues raised in the acceptability study were intimately related to mothers' general infant feeding experiences within the community. In their narratives, mothers provided rich constructs of the interrelationship between personal infant feeding experiences, health messages and disclosure of HIV status. Therefore, the emergent data address a different question from the acceptability study: here, the research question seeks to explore challenges and factors enabling safe infant feeding practices within an HIV-prevalent, resource-poor setting.

\section{Methods}

The present study was set in an urban informal settlement in the province of KwaZulu-Natal, South Africa. KwaZuluNatal has the highest antenatal HIV burden in the country, with current prevalence estimated at $39 \cdot 1 \%{ }^{(13)}$. It is a setting in which AFASS conditions rarely exist and, hence, safe breast-feeding practices are particularly important.

Participant recruitment and interviews took place from October 2004 to March 2005, and were conducted by a trained community research partner in collaboration with the researcher (L.S.). The community research partner was a resident of the study community, while the researcher has a dietitian/nutritionist and lactation consultant professional background. Our sample of mothers of young infants was purposefully selected, being recruited both at the local clinic as well as within the community. In the community, participants were identified by approaching households with infant laundry hanging in the yard. The mean age of participating mothers ( $n$ 11) was 25 years, with the average age of the index infant being 3 months. Serostatus was not a selection criterion for study participants.

Sample selection for the larger acceptability study recruited the mothers as a starting point and, via the snowball technique, recruited a total of thirty-one participants including family members such as partners, grandmothers and mothers-in-law, health-care workers, lay counsellors, traditional healers and child-care professionals. Sample size was based on reaching the point of saturation, where no new emergent issues were identified with additional participant interviews. Data presented in the current paper are principally from the mothers participating in the original acceptability study.

Once the purpose of the study was explained and consent given, interviews were conducted in isi-Zulu utilizing Patton's ${ }^{(14)}$ general interview guide approach which advocates an open-ended conversational interview format.

Data collection was achieved through tape recording of in-depth interviews plus notes made during and shortly following interviews. All interviews were transcribed and then translated into English by the community research partner and researcher.

Text data from transcripts and notes were evaluated using the content analysis method ${ }^{(15)}$, whereby codes were developed based on repeat comments, concepts and keywords identified from the text following several readings of a few transcripts. These codes were used in categorizing concepts in the remaining transcripts, with new codes developed when new words or concepts emerged. Codes were finally streamlined into thematic subcategories. The process was conducted independently by the researcher and research assistant (S.N.) and agreement reached 
through discussions. Confirmation of interpretation of data was achieved through member checks with mothers, clinic and community participants, where possible.

Notes resulting from observations and informal conversations within the community and at the clinic were used as a source of additional data (triangulation) to help validate our findings and to establish trustworthiness of the data ${ }^{(16)}$.

Ethics approval was obtained from the University of KwaZulu-Natal and McGill University.

\section{Results}

The majority of the mothers identified themselves as HIVinfected. The feeding practices of the eleven mothers comprised four formula feeders, five mixed feeders and two mothers who exclusively breast-fed. Only one of the four mothers who chose to formula feed was able to practise exclusive formula feeding (EFF) of her 3-month-old infant. Factors enabling the one mother who practised EFF included being employed, use of the free formula programme and disclosure of her HIV status to her partner. None of the other three mothers disclosed their status, but reported a reliance on family and the free formula programme as their sources of formula. These mothers reported formula supply to be inconsistent, which facilitated inappropriate feeding (use of cow's milk or other fluids) including over-dilution of formula.

The process to access the free formula for a 6-month period from the clinic (based on our observations and conversations with staff and clients) required women living with HIV to identify themselves at the clinic. Free formula is not available immediately (up to 1 month waiting period), so that mothers initially require an alternative source of formula allotment.

Mothers who were practising mixed feeding added other fluids and/or small quantities of foods to breast feeds. Asked why they fed in this manner, two reported being advised to do so by family members while another indicated she mixed fed to promote infant satiety and night sleeping. The remaining two mothers reported being directed to formula feed following delivery at the local hospital because of their positive HIV status. However, both mothers began mixed feeding following discharge from hospital: the first mother added breast-feeding to the formula feeds, motivated by her partner and her own desire to breast-feed. The second mother reported not having access to formula, she instead breast-fed and added diluted solids, a practice based on past experiences and her perception that breast milk was insufficient to satisfy her newborn.

Only two mothers were practising EBF, one of the mothers described the events leading to her breast-feeding exclusively:

I saw that my baby was not full from my milk when

I got home from the hospital. So I went out and got

a small tin of milk [formula] and gripe water, because
Table 2 Maternal constructs of key influences on infant feeding practices

Stigma and disclosure

It was hard to tell the baby's father, he was angry, then quiet but now he really wants the baby to be ok. I want him to test himself, but we will see what happens.

(Mother disclosed to partner; practising EBF)

\section{Confusion and coercion}

At the clinic we always talked about how I can breast-feed my baby, but at the hospital they said no I should use the tin milk... that is what I do, but I have to worry about always having it. (Mother who wants to disclose to sister; practising MF)

Diarrhoea, sickness and free formula

I worry about diarrhoea with the baby, but now she is ok, she had some last time, it went away I used some muthi [traditional medication].

(Mother disclosed to partner and friend; practising EFF)

I have some formula from the clinic, I hope it lasts me because I have to wait till the next month to get more... and she is growing and hungry.

(Mother who has not disclosed - hides use of formula; practising MF)

EBF, exclusive breast-feeding; EFF, exclusive formula feeding; MF, mixed feeding.

he was crying at night. But when I took the baby to the clinic, they told me I cannot mix feed. They explained that the baby is satisfied with my breast milk. So I continued just breast-feeding and I can see I have enough milk and my baby is ok. My partner is ok with me only breast-feeding because he knows my problem [seropositive status]. People try to feed the baby, they shout at me and ask me why I only breast-feed? I told them if they give food to my baby, the baby will be sick. I am not going to visit my family in the farm [rural area] until my baby is 6 months, at that time the baby will be eating food.

The second mother had been breast-feeding her 4-month-old exclusively since birth. Her initial motivation to EBF was as a result of viewing a breast-feeding promotion video at the antenatal clinic. This mother lived with her own mother and grandmother who were both very supportive of $\mathrm{EBF}$, with the grandmother informing us she cup-fed expressed breast milk made available by the infant's mother when she was not home. Both relatives attributed their support of EBF to their awareness of the mother's positive serostatus, her sharing of advice and information given by counsellors at the clinic as well as their witnessing healthy growth of the infant.

Table 2 summarizes maternal constructs of key influences on infant feeding practices, emergent from interviews with mothers as they contextualized their feeding experiences.

\section{Stigma and disclosure of HIV status}

In their discussions of feeding practices, most mothers singled out stigma and the challenge of disclosure as 
significant considerations in their infant feeding decisionmaking process. It was clear from our discussions that fear of reprisal from family members or friends, as well as from the community at large, can serve as a barrier for mothers to disclose their HIV status. Mothers consistently informed us that in many instances lack of disclosure and the concomitant lack of support may lead some women to follow practices they do not necessarily agree with. One mother pointed out:

If you have a partner who doesn't know your status it's a big problem with breast-feeding for a mother. The father wants to buy formula because he thinks our baby needs tin milk and breast-feeding [to be well fed], which the counsellor said I should not do because my milk is enough... I know, but what can I do?

This climate of HIV stigma and discrimination at times contributed to some mothers being fearful of exposure when they picked up their formula supply from public spaces, such as a clinic. In the same manner, within the home, a mother feeding formula might hide the infant feeding bottle from specific individuals, giving instead the impression she was breast-feeding. As was the experience of one mother:

I fed the baby before she [relative] came and then when we sat and talked I put the baby near my breast, so that she thinks I breast-feed the baby.

Mothers who had disclosed their HIV status were less concerned with stigma and often felt they had support to feed their infants according to their intentions.

\section{Confusion and coercion}

Emerging from our conversations was the confusion and anxiety that infant feeding in the context of HIV elicited for the mothers. Feeding newborns presents mothers with much conflict that relates to both infant- and maternalfocused concerns. On the one hand, if a seropositive mother wishes to breast-feed, she grapples with her fear of infecting her infant; conversely, were she to choose to formula feed she runs the risk of arousing suspicion regarding her HIV status. Additionally, in many instances, use of formula, particularly in early infancy, is often in disagreement with a partner or family member's feeding expectations:

But now I did not know what to do because I did not want to give this sickness [HIV] to my child, but my mother and the father [of the child] would ask me why I was not breast-feeding this child.

(Mother)

Most mothers did not recall receiving informative infant feeding counselling prior to the birth of their child; instead their experiences consisted of being recipients of inconsistent and confusing advice and direction from various health-care workers at the delivery of their newborn. Some mothers shared their experiences of being directed by hospital staff to feed formula, with breast-feeding strongly discouraged. This directive was given in the absence of an assessment of the mother's ability to feed formula safely. Mothers reported being cautioned by staff that to breast-feed was equivalent to transmitting the virus to their infants, which they were told was unnecessary since the government provided free formula for use by infected mothers. None of the mothers advised to formula feed recalled hospital staff providing counselling on how to use formula appropriately (i.e. EFF, not adding solids and foods to the diet for infants below 6 months of age, appropriate formula preparation).

This coercion to formula feed served as a source of confusion, since the directive was in conflict with messages indicating EBF as a viable option for HIV-infected mothers:

The nurse told me that those who say I can breastfeed are playing with me; she said I will infect my child using my own milk. I should be a good mother and use the baby tin milk [formula].

(Mother)

Mothers directed to use the free formula programme were quick to point out that the programme presented its own problems, the primary one being sustainability:

I was afraid to breast-feed because of other things ... this sickness [HIV]; but the tin milk cost too much and the nurses at the clinic sometimes tell us they have no milk to give us.

(Mother)

This mother's reaction to the unavailability of formula was to engage in inappropriate feeding of her young infant, using sugar water plus solid food, until the clinic received the formula supply.

Coercion was not limited to health-care workers, but also came from other sources:

Sometimes girls do not breast-feed because her partner says that their child only drinks baby milk [formula]. But sometimes the partner is not around, so she borrows milk to feed the baby until the father comes back. But what happens if the father disappears... then you cannot pay that baby milk back, you have a hungry crying baby with no food and the father who is long gone.*

(Counsellor)

Given the level of pressure some infected mothers received to use formula, we were interested in the experiences of women who were able to resist yielding to this pressure. These were women who still initiated

\footnotetext{
* A partner who disappears is of concern since some mothers are dependent on their partners for financial support.
} 
breast-feeding despite being counselled to formula feed, mostly motivated by a fear of scrutiny from family members and friends as a result of not breast-feeding. Health benefits and affordability were secondary considerations in choosing to breast-feed:

Babies feeding from the breast grow well and look big and healthy... but really, nobody asks funny questions when you breast-feed.

(Mother)

These were also the mothers who ended up mixed feeding. The two mothers practising EBF, although both felt pressured in the hospital to use formula, reported a strong commitment to their prenatal decision to EBF (even though one mother used formula briefly due to infant satiety concerns) and felt supported by family to do so.

\section{Diarrboea, sickness and free formula}

Infant-centred concerns focusing on morbidity issues emerged as an area readily discussed by almost all participants interviewed. Diarrhoea was the most commonly cited child illness of concern:

Now that I am not breast-feeding I am worried; my other babies had my milk and there was no diarrhoea, not like my sister-in-law, she works and she buys tin milk for her children and they had a lot of diarrhoea... I know, I used to see them.

(Mother)

Both counsellors and community health workers reported that they had observed an increased presentation at the clinic of children suffering from malnutrition, failure to thrive, diarrhoea and dehydration. They felt that the increase in these morbidities occurred shortly after the availability of free formula. Prior to the introduction of the programme there was a stronger commitment from both health-care workers and mothers to breast-feeding; as a result there were more women breast-feeding in the community and, in their opinion, less of these childhood morbidities. This observation was confirmed through discussions with other health-care workers at the community clinic:

First we were happy with the free formula because we thought our problems with the infected mothers were over... But now we see it is trouble, really it is a lot of trouble for the baby... diarrhoea, sickness and not growing well.

\section{(Health-care worker)}

Related to formula use, workers also spoke of an increased frequency of mothers presenting at the clinic with infants who were sick as a result of being fed sugar water for an extended period. One counsellor recounted her experience of working with a mother who had fed sugar water to her infant in excess of a month, as a result of not having formula in the house. The child presented with diarrhoea and was severely dehydrated; however, the mother was unaware of her error since she had observed the infants in hospital routinely fed sugar water. This was not an isolated incident; staff at day-care centres and traditional healers all relayed similar encounters with mothers who chose not to breast-feed but yet had no access to appropriate breast-milk substitutes.

One mother provided a poignant example of the complexities of access to free formula for families with competing needs:

Many mothers sell the tin milk from the clinic...

because you have to have food in the house.

\section{Discussion}

Lack of disclosure of HIV status as a result of existing stigma and discrimination associated with HIV-seropositive status emerged as a key factor in the infant feeding decision-making process. For infected mothers, their serostatus added a level of complexity to feeding their newborns. In formulating infant feeding decisions, women enter a risk-benefit type of dilemma, caught between a desire to feed their child in a manner that prevents infection, with a need to optimize infant health, while respecting feeding expectations of partners and family members. These factors interact against a backdrop of cultural norms that value breast-feeding but remain discriminatory towards HIV-infected members of society. Although most infant feeding strategies render women the bearers of responsibility for feeding their children, practices are rarely the sole decision of the mother. As a result, mothers identified two factors that operate synergistically to facilitate optimal feeding. The first involves direct and voluntary disclosure (Table 1) of the mother's HIV status to an identified individual, with receiving support viewed as an important second critical factor. Through disclosure the mother actively shares, with an identified support person, what she may perceive as the risk (i.e. possible infection of an infant and or exposure to stigma) associated with the selected feeding practice. Conceptually, as illustrated in Fig. 1, disclosure and support often facilitate a supportive connection which enables women to practise optimal infant feeding. This relationship is particularly beneficial for women who choose to breast-feed, since it optimizes the opportunity for mothers to practise EBF. Indeed, Varga et $a l^{(12)}$ found that women who disclosed their HIV status did so motivated by a need for support of their feeding choices and practices. Our data confirm this finding. Although disclosure seemed to also facilitate EFF, an additional prerequisite for safe feeding requires AFASS conditions, a rare occurrence for families living within resource-poor communities. Access to free formula seems not to solve the issue. Although at a policy level free formula may seem theoretically to be an enabling factor, in reality it is unsustainable, as indicted by our findings. 


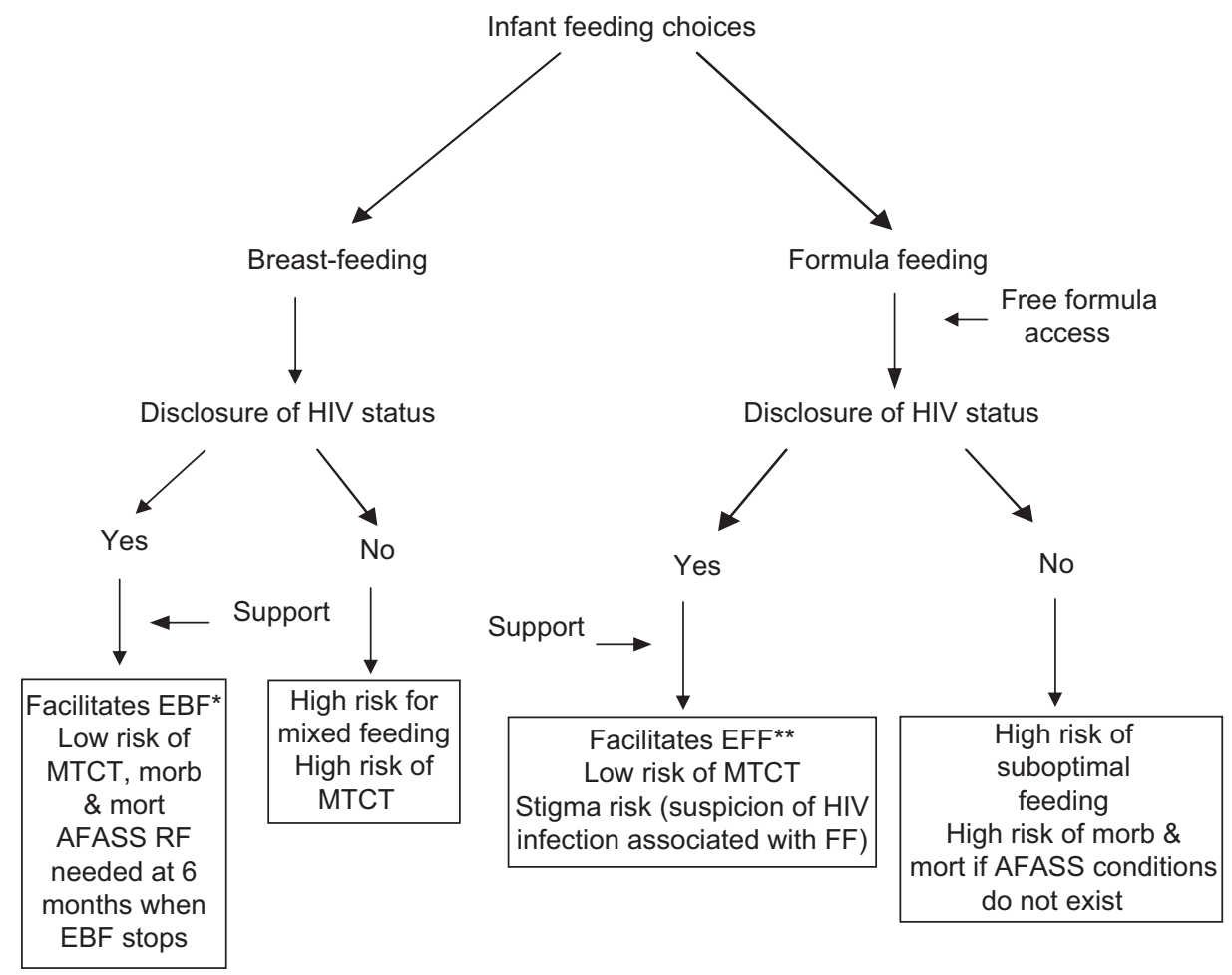

Fig. 1 Theoretical relationship between disclosure and support. EBF, exclusive breast-feeding; EFF, exclusive formula feeding; FF, formula feeding; MTCT, mother-to-child transmission; AFASS, affordable, feasible, acceptable, safe and sustainable; RF, replacement feeding; morb, infant morbidity (i.e. diarrhoea, malnutrition); mort, mortality. *Optimal breast-feeding, recommended for settings without AFASS conditions; ${ }^{* *}$ optimal formula feeding, not recommended for settings without AFASS conditions

Translation of international infant feeding guidelines into national policies and ultimately into PMTCT programmes requires evidence-based tools that facilitate operational implementation of the recommendations. The AFASS conditions act as such a tool, developed to guide PMTCT programmes to maximize HIV-free child survival $^{(10)}$. Given that lack of disclosure acts as a significant barrier to adherence to PMTCT programmes ${ }^{(17)}$, understanding ways to address disclosure plays a crucial role in promoting optimal infant feeding. Identified barriers to disclosure include fear of violence, accusations of infidelity, discrimination and abandonment ${ }^{(17)}$. Despite these barriers, women who do disclose their status report increased social support ${ }^{(18)}$. Conceptually, disclosure of HIV status to a support person may create an environment where optimal feeding is accepted, enabling mothers to feed infants within their economic means.

Our findings indicate that among mothers who had not disclosed their status, mixed feeding, which carries the highest risk for transmission ${ }^{(8,10)}$, was a more common practice. Clearly, lack of maternal disclosure of seropositive status seems to pose a significant risk to infant health via poor feeding practices.

The type of disclosure seems to also be relevant, such that the risk of indirect and involuntary disclosure (Table 1) meant women were often careful that their actions (i.e. EFF) did not imply positive HIV status. Moreover, mothers who had not disclosed their status engaged in creative explanations of their feeding behaviours to avoid disclosure by proxy.

In the developing world, breast milk is an affordable, safe and sustainable food that plays a critical role in child growth and development. Within resource-poor settings, absence of AFASS conditions precludes use of replacement feedings such as formula. Safe breast-feeding practices are therefore critical for promoting HIV-free infant survival of children born to infected mothers in the developing world. Policies and guidelines that discourage breast-feeding by HIV-infected African mothers ignore the complex interrelationship between emotional, social, cultural and financial constraints faced by women in most of sub-Saharan Africa, where two-thirds of the world's HIV-infected persons live. Although policies supporting international guidelines do not ensure practice, within our study setting, where conditions of poverty prevail and HIV prevalence is high, infant feeding practices of mothers were contrary to national guidelines. The nature of encounters mothers experienced with health-care workers contributed to the existing polarization between recommendations and actual practices. A disturbing finding was the coercion mothers encountered from hospital staff following delivery, where mothers were directed to use formula as a means of avoiding vertical transmission of HIV. In these situations, no consideration 
was given to the personal desires or living conditions of the mother.

Owing to unclear messages, mothers in our study seemed to be unaware of the dangers associated with mixed feeding, confirming the findings of others ${ }^{(19)}$. Doherty et $a l^{(20)}$ also showed that international guidelines were poorly implemented in their cohort study across three PMTCT sites in South Africa. Mixed feeding has been consistently shown to be a highly risky feeding pattern for infants born to HIV-infected mothers ${ }^{(6,21)}$, and most recently was found to double the risk of infection of infants fed formula plus breast milk compared with their EBF counterparts. The risk of HIV transmission increased eleven-fold when solids were added to breast-feeding ${ }^{(8)}$. Much frustration seems to exist from the counsellors' perspective, where health-care workers feel overwhelmed with their work load and experience significant time constraints, resulting in more directive counselling ${ }^{(22)}$. Confusion, misconceptions and personal biases towards feeding options $^{(23)}$ have also been an issue, as has an overall sense of being poorly trained which has been perceived by health-care staff as undermining their authority ${ }^{(24)}$. Clearly, issues of mothers feeling judged and shamed coupled with health-care workers experiencing stress, depression and burn-out ${ }^{(25)}$ will continue to increase the gap between sound policy and infant feeding practices.

Infant health emerged as an issue of great concern among most participants, both from the perspective of HIV infection risk but also related to diarrhoea and malnutrition morbidities, which were thought to be on the rise in the community. Food insecurity and poor living conditions are identified as underlying contributing factors to these child morbidities. Promotion, protection and support of breast-feeding continues to be the suggested strategy to address these morbidity and mortality risks, even in the era of HIV $^{(26)}$. However, in our resource-poor study setting, breast-feeding, a secure source of infant food, seemed to be under threat of declining according to mothers and other informants, including health-care workers. This trend in some areas of Africa has been attributed to both maternal perceptions, such as breast milk insufficiency, as well as increased pressure to use formula $^{(27)}$. In our study, the decline in breast-feeding was believed to be as a consequence of the availability of a free formula programme. Given the reported lack of consistent availability and the insufficiency of the supplied allotment, there is no evidence to support a free formula policy for mothers living with HIV within an African setting. Such an undertaking ignores the hidden costs associated with appropriate formula feeding while promoting mixed feeding, among other hazards ${ }^{(28)}$. Recent events in Botswana, a well-resourced country, illustrate the unacceptable risks associated with free formula available to HIV-infected women ${ }^{(29)}$. Increased presence of formula serves as a risk for 'spillage' of formula use into the non-infected or unknown HIV status populations. As a result there can be a significantly higher risk of illness and death of infants and young children in these communities, as was evidenced in the Botswana case, where even the HIV-uninfected and unexposed infants experienced greater diarrhoeal-related morbidity and mortality ${ }^{(30)}$.

The current study was limited by its size of eleven mothers (plus an additional twenty family and community members in the larger acceptability study). However, sampling was stopped because of similarity of information provided by participants (saturation point). Studying one community serves as an additional limitation, although the community was felt to be representative of settings with a very high prevalence of HIV infection and similar to South African settings with limited economic resources. As community trust was essential to establish in order to be able to undertake our study, our focus was placed on one community.

\section{Conclusion}

Mothers bear the responsibility of feeding infants, but decisions on practices are often not made in isolation. Hence, infant feeding strategies can be viewed as risky and divergent from cultural and social norms for these women. However, this challenge does not preclude the promotion of safe breast-feeding practices, since support and voluntary and direct disclosure of HIV status greatly facilitates the uptake of optimal feeding practices. Mothers who disclosed their status and secured support were able to EBF, a recommended practice for improving HIV-free infant survival. On the other hand, inappropriate infant feeding advice resulted in creating confusion and promoting suboptimal practices such as mixed feeding, an HIV transmission risk. Therefore, there is an urgent need for health-care services to invest in ongoing highquality training programmes that provide ongoing support for health workers to counsel mothers in a manner that enables evidence-based optimal infant feeding. Additionally, training should include facilitation of HIV status disclosure.

\section{Acknowledgements}

We received funding from the Canadian Institute of Health Research (CIHR) and from the International Development Research Centre (IDRC), Ottawa, Canada. There is no conflict of interest associated with the research. L.S. developed the study concept and study design, conducted recruitment of participants, data collection, data analysis and data interpretation, and took the lead in manuscript preparation. A.C. collaborated in the study conception and study design, and assisted in recruitment, data interpretation and manuscript preparation. S.N. conducted participant recruitment and data collection, 
and participated in interpretation and manuscript preparation. K.G.-D. participated in the study conception and development, study design, data interpretation and manuscript preparation.

\section{References}

1. Joint United Nations Programme on HIV/AIDS (2008) Report on the Global AIDS Epidemic. Geneva: WHO/ UNAIDS.

2. Joint United Nations Programme on HIV/AIDS (2006) Report on the Global AIDS Epidemic. Geneva: WHO/ UNAIDS.

3. De Cock KM, Fowler MG, Mercier E, de Vincenzi I, Saba J, Hoff E, Alnwick DJ, Rogers M \& Shaffer N (2000) Prevention of mother-to-child HIV transmission in resource-poor countries: translating research into policy and practice. JAMA 283, 1175-1182.

4. Jones G, Steketee RW, Black RE, Bhutta ZA \& Morris SS (2003) How many child deaths can we prevent this year? Lancet 362, 65-71.

5. Coutsoudis A, Pillay K, Kuhn L, Spooner E, Tsai WY \& Coovadia HM (2001) Method of feeding and transmission of HIV-1 from mothers to children by 15 months of age: prospective cohort study from Durban, South Africa. AIDS 15, 379-387.

6. Iliff PJ, Piwoz EG, Tavengwa NV, Zunguza CD, Marinda ET, Nathoo KJ, Moulton LH, Ward BJ \& Humphrey JH; ZVITAMBO Study Group (2005) Early exclusive breastfeeding reduces the risk of postnatal HIV-1 transmission and increases HIV-free survival. AIDS 19, 699-708.

7. Newell M (2006) Current issues in the prevention of mother-to-child transmission of HIV-1 infection. Trans $R$ Soc Trop Med Hyg 100, 1-5.

8. Coovadia H, Rollins N, Bland R, Little K, Coutsoudis A, Bennish M \& Newell ML (2007) Mother-to-child transmission of HIV-1 infection during exclusive breastfeeding in the first 6 months of life: an intervention cohort study. Lancet 369, 1107-1116.

9. World Health Organization/Joint United Nations Programme on HIV and AIDS/UNICEF (1998) Technical Consultation on HIV and Infant Feeding. Geneva: WHO.

10. World Health Organization (2006) WHO HIV and Infant Feeding Technical Consultation Held on behalf of the Interagency Task Team (IATT) on Prevention of HIV Infections in Pregnant Women, Mothers and their Infants, Geneva, October 25-27, 2006. Consensus Statement. http://who.int/reproductivehealth/stis/mtct/infantfeedingconsensusstatement.pdf (accessed January 2009).

11. Rutenberg N (2003) HIV testing and disclosure: experiences from prevention of mother- to- child transmission programs in Kenya and Zambia. In Women's Experiences with HIV Serodisclosure in Africa: Implementation for VCT and PMTCT. Report of a USAID Technical Meeting, pp. 7-11. Washington, DC: USAID Office of HIV/AIDS.

12. Varga CA, Sherman GG \& Jones SA (2006) HIV-disclosure in the context of vertical transmission: HIV-positive mothers in Johannesburg, South Africa. AIDS Care 18, 952-960.

13. Department of Health (2007) National HIV and syphilis antenatal sero-pravlence survey in South Africa, 2006. http:// www.doh.gov.za/docs/hivaids (accessed January 2007).
14. Patton MQ (2002) Qualitative Research and Evaluation Methods. Thousand Oaks, CA: Sage.

15. Cavanagh S (1997) Content analysis: concepts, methods and applications. Nurse Res $\mathbf{4}$, issue 3, 5-16.

16. Lincoln Y \& Guba E (1985) Naturalistic Inquiry. New York: Sage.

17. Medley A, Garcia-Moreno C, McGill S \& Maman S (2004) Rates, barriers and outcomes of HIV serostatus disclosure among women in developing countries: implications for prevention of mother-to-child-transmission programmes. Bull World Health Organ 82, 299-307.

18. Matthews C, Kuhn L, Fransman D, Hussey G \& Dikweni L (1999) Disclosure of HIV status and its consequences. $S$ Afr Med J 89, 1238

19. Chopra M, Doherty T, Jackson D \& Ashworth A (2005) Preventing HIV transmission to children: quality of counseling of mothers in South Africa. Acta Paediatr 94, 357-363.

20. Doherty T, Chopra M, Jackson D, Colvin M \& Persson LA (2007) Effectiveness of the WHO/UNICEF guidelines on infant feeding for HIV-positive women: results from a prospective cohort study in South Africa. AIDS 21, 1791-1797.

21. Coutsoudis A, Pillay K, Spooner E, Kuhn L \& Coovadia HM (1999) Influence of infant-feeding patterns on early mother-to-child transmission of HIV-1 in Durban, South Africa: a prospective cohort study. Lancet 354, 471-476.

22. di Paoli M, Manongi R \& Klepp KI (2002) Counsellors' perspectives on antenatal HIV testing and infant feeding dilemmas facing women with HIV in northern Tanzania. Reprod Health Matters 10, 144-156.

23. Piwoz E, Ross J \& Humphrey J (2004) Human immunodeficiency virus transmission during breastfeeding: knowledge, gaps, and challenges for the future. Adv Exp Med Biol 554, 195-210.

24. Leshabari SC, Blystad A, de Paoli M \& Moland KM (2007) HIV and infant feeding counselling: challenges faced by nurse-counsellors in northern Tanzania. Hum Resour Health 5, 18; available at http://www.human-resourceshealth.com/content/5/1/18

25. Buskens I \& Jaffe A (2008) Demotivating infant feeding counselling encounters in southern Africa: do counsellors need more or different training? AIDS Care 20, 337-345.

26. Bourne LT, Hendricks MK, Marais D \& Eley B (2007) Addressing malnutrition in young children in South Africa. Setting the national context for paediatric food-based dietary guidelines. Matern Child Nutr 3, 230-238.

27. Walker AR \& Adam FI (2000) Breastfeeding in sub-Saharan Africa: outlook for 2000. Public Health Nutr 3, 285-292.

28. Coutsoudis A, Goga AE, Rollins N \& Coovadia HM (2002) Free formula milk for infants of HIV-infected women: blessing or curse? Health Policy Plan 17, 154-160.

29. Creek T, Luo C \& Quick T (2006) HIV-exposed children highly affected by deadly outbreak of diarrhea and severe acute malnutrition - Botswana, 2006. Presented at The 2006 HIV/AIDS Implementers Meeting of the President's Emergency Plan for AIDS Relief, Durban, South Africa, 12-15 June 2006.

30. Creek T, Arvelo W, Kim A, Lu L, Bowen A, Mach O, Finkbeiner T, Zaks L, Masunge J \& Davis M (2007) A large outbreak of diarrhea among non-breastfed children in Botswana 2006 - implications for HIV prevention strategies and child health. Presented at XIV Conference on Retroviruses and Opportunistic Infections, Los Angeles, CA, USA, 25-28 February 2007. 